\title{
Miniaturization of Patch Antenna Using Metasurface
}

\author{
H.L. Zhu, S.W. Cheung and T.I. Yuk
}

\begin{abstract}
In this letter, it is proposed to use metasurface (MS) to miniaturize patch antenna. The MS studied is composed of two layers, layers 1 and 2. Layer 1 has simple rectangular microstrip lines as unit cells and layer 2 has the complimentary pattern of layer 1 . The MS is placed on top of and in direct contact with a small patch antenna, forming a miniaturized-patch antenna operating at 4 GHz. For comparison, another patch antenna without using MS is designed to operate at the same frequency. Both antennas are studied using computer simulation and measurement. Results show, with the use of the MS, the patch antenna can have the size reduced by $67 \%$, yet achieving a similar performance. The simulated and measured results in reflection coefficients, efficiencies, realized peak gains and radiation patterns of the antennas are presented. The cable effects caused by the feeding cable used in measurement are studied.
\end{abstract}

Keywords: miniaturization, patch, metasurface

\section{Introduction}

With the increasing demand for compact wireless devices in recent year, small efficient antennas with simple structures have been a topic of great interests [1-8]. Due to the simple and low profile structure, planar antenna seems to be a good choice for many portable wireless devices. However, antenna size is in general inversely proportional to the operating frequency. For patch antenna, the size of the radiator alone is normally $0.5 \lambda_{0} \times 0.4 \lambda_{0}$, where $\lambda_{0}$ is the wavelength at the operating frequency $[9,10]$. With the feed line and ground plane taken in account, the overall size is larger and may not be small enough to fit into small wireless devices. Thus a lot of efforts have been made to miniaturize the size of planar antennas using various methods [1-8].

Metasurface (MS) is a 2D version of metamaterial [11] and has succinct surface structure with low profile, so it can be combined with planar antennas such as planar patch, slot and monopole antennas for performance enhancements, yet retaining the low profile structures. For example, it was shown in [12-18] that the performances of patch antennas could be enhanced significantly by placing a MS atop of it. In general, the size of patch antenna is mainly determined by the lowest resonant frequency, i.e. the larger the size of the patch antenna, the lower is the resonant frequency. Thus if we can shift down the resonant frequency, the patch antenna can be miniaturized.

In this letter, it is proposed to use MS to miniaturize patch antennas. A simple patch antenna is designed on a double-sided substrate, having an area of $0.27 \lambda_{0} \times 0.27 \lambda_{0} \times 0.03 \lambda_{0}$, where $\lambda_{0}$ is the wavelength at the operating frequency in free space. The MS is composed of two layers, layers 1 and 2, each designed on a single-sided substrate. Layer 1 has simple rectangular microstrip lines as unit cells and layer 2 has the complementary pattern of layer 1 . The MS is placed on top of and in direct contact with the patch antenna for miniaturization. Another patch antenna is designed to have a similar performance at the same frequency without using MS for comparison. The two 
antennas are studied using the EM simulation tool CST and measured using the antenna measurement equipment Satimo Starlab system. Results shows that, with the use of the MS, the patch antenna can reduce the size by $67 \%$, yet having a similar performance in terms of reflection coefficient, efficiency and realized boresight gain. The cable effects caused by the feeding cable used in measurement are also studied and used to explain the discrepancies between the simulated and measured results

\section{Designs of Antenna and Metasurface}

The patch antenna used for studies here is designed on a double-sided substrate as shown in Fig. 1 (a) which consists of a patch with microstrip-fed using a $50-\Omega$ feed line on one side and a ground plane on the other side. The feed line is fed using a SMA connector on the ground plane through a via on the substrate. The patch has a length of $P_{w}=10 \mathrm{~mm}$ which, together with the MS designed, generates a frequency band at about $4 \mathrm{GHz}$. The patch antenna occupies a total area of $T \times T=20 \times 20 \mathrm{~mm}^{2}=400 \mathrm{~mm}^{2}$ and is known here as patch 1 .

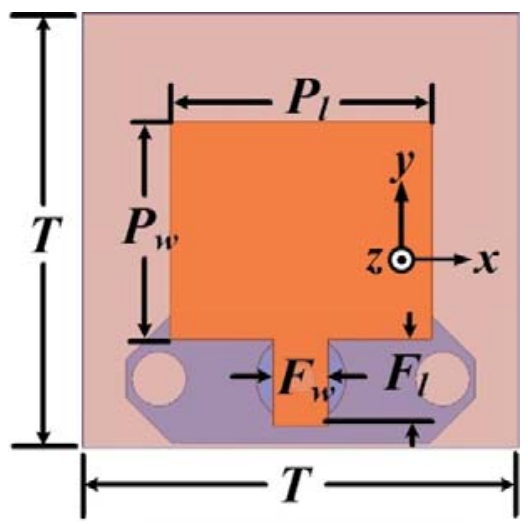

(a)

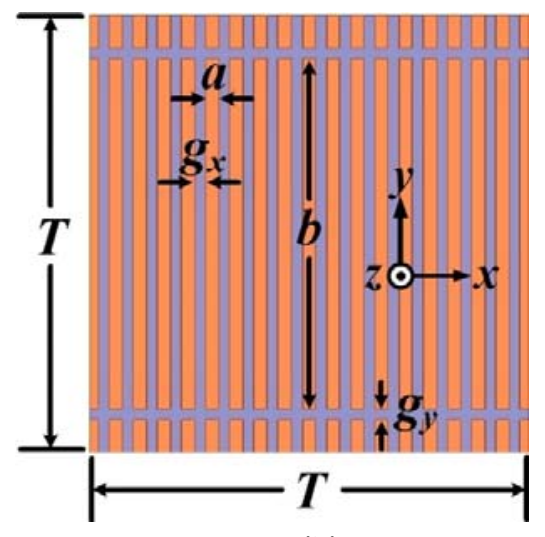

(b)

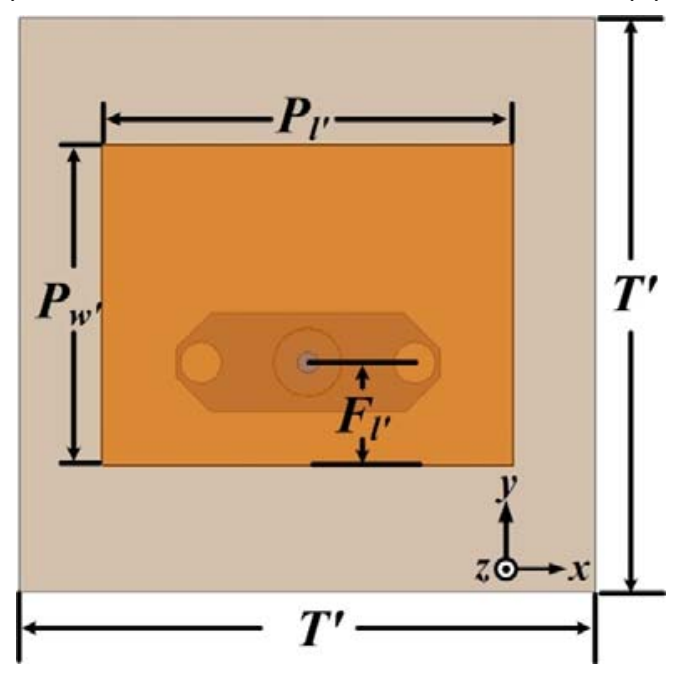

(c)

Fig. 1. Geometries of (a) patch 1 , (b) metasurface (MS) and (c) patch 2. 
The metasurface (MS) used in our miniaturized-patch antenna has two layers, layers 1 and 2, both designed on a single-sided substrate. Layer 1 is composed of unit cells having microstrip lines with width $a$ and length $b$ as shown in Fig. $1(b)$. The unit cells are printed periodically along the $x$-axis and $y$-axis directions on the $x$-y plane. The pattern on layer 2, as shown in Fig. 2, is just the exact complementary pattern of layer 1 . The miniaturized-patch antenna is assembled by placing the non-copper side of layer 1 in direct contact with the radiator of the patch antenna, and the non-copper side of layer 2 in direct contact with the copper-side of layer 1 as shown in Fig. 2. This leads to a very compact and low profile structure.

The antenna together with the SMA connector as shown in Fig. 2 is studied and designed using the EM simulation tool CST on a Rogers substrate, RO4350B, with a dielectric constant of 3.5, a loss tangent of 0.004 and a thickness of $0.762 \mathrm{~mm}$. For the purpose of illustration, the miniaturized-patch antenna is designed to operate at the frequency of $4 \mathrm{GHz}$. The final dimensions of the patch antenna are listed in Table I. The miniaturized-patch antenna occupies a total area of $20 \times 20 \mathrm{~mm}^{2}=400 \mathrm{~mm}^{2}$, same as that of patch 1 .

To study size reduction of the miniaturized-patch antenna using MS, another patch antenna, known here as patch 2, without using the MS is designed, as shown in Fig. 1(c), to have the same resonant frequency, bandwidth and realized boresight gain as those of the miniaturized-patch antenna. The dimensions of patch 2 are listed in Table II. Patch 2 is used as a reference antenna for size comparison. It can be seen that patch 2 with an area of $35 \times 35 \mathrm{~mm}^{2}=1225 \mathrm{~mm}^{2}$ is much larger than patch 1 with an area of $20 \times 20 \mathrm{~mm}^{2}=400 \mathrm{~mm}^{2}$. The MS reduce the required size of the patch antenna by $67 \%$.

TABLE I. DIMENSIONS OF PATCH 1 AND MS (UNIT:MM)

\begin{tabular}{|c|c|c|c|c|c|c|c|c|}
\hline $\boldsymbol{P}_{l}$ & $\boldsymbol{P}_{\boldsymbol{w}}$ & $\boldsymbol{F}_{\boldsymbol{w}}$ & $\boldsymbol{F}_{\boldsymbol{l}}$ & $\boldsymbol{a}$ & $\boldsymbol{B}$ & $\boldsymbol{g}_{\boldsymbol{x}}$ & $\boldsymbol{g}_{\boldsymbol{y}}$ & $\boldsymbol{T}$ \\
\hline 12 & 10 & 2.5 & 6 & 0.6 & 16 & 0.5 & 0.5 & 20 \\
\hline
\end{tabular}

TABLE. II DIMENSIONS OF PATCH 2 (UNIT:MM)

\begin{tabular}{|c|c|c|c|}
\hline $\boldsymbol{P}_{l^{\prime}}$ & $\boldsymbol{P}_{\boldsymbol{w}^{\prime}}$ & $\boldsymbol{T}^{\prime}$ & $\boldsymbol{F}_{\boldsymbol{I}^{\prime}}$ \\
\hline 25 & 19.2 & 35 & 3.5 \\
\hline
\end{tabular}

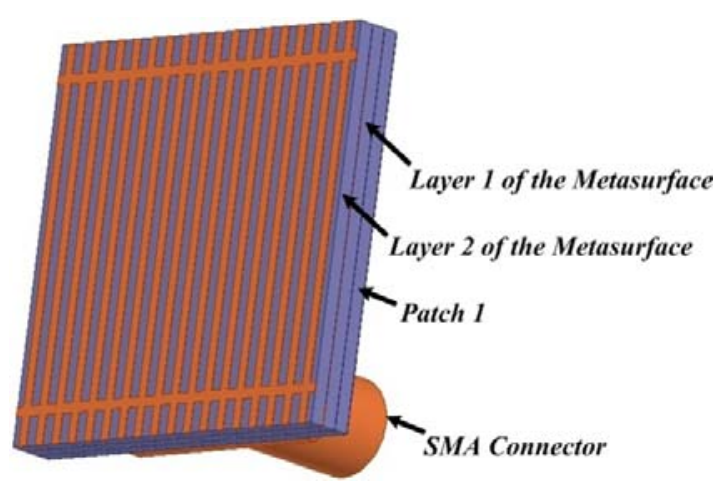

Fig. 2. Minaturized-patch antenna using MS.

The dimensions in Table 1 are used to fabricate patch 1 and the MS as shown in Fig. 3 . The minaturised patch antenna is then assembled, as shown in Figs. 4, using patch 1 and the MS. Our antenna measurement equipment, Satimo Starlabe system, is used to measure the performaces 
of the minaturized patch antenna, patches 1 and 2 for comparison. The measured results are also compared with simulated results.

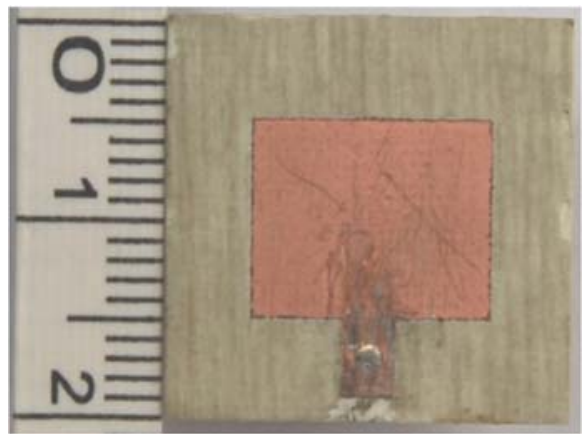

(a)

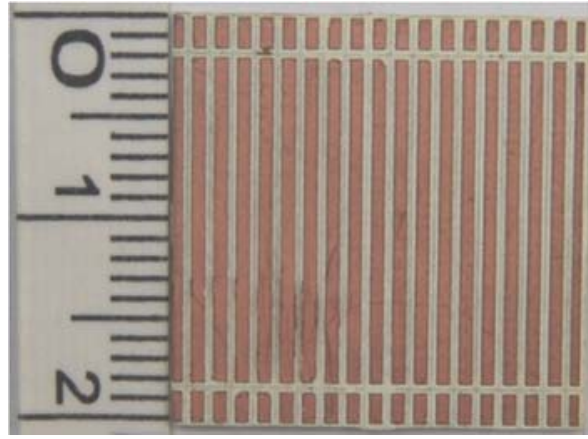

(c)

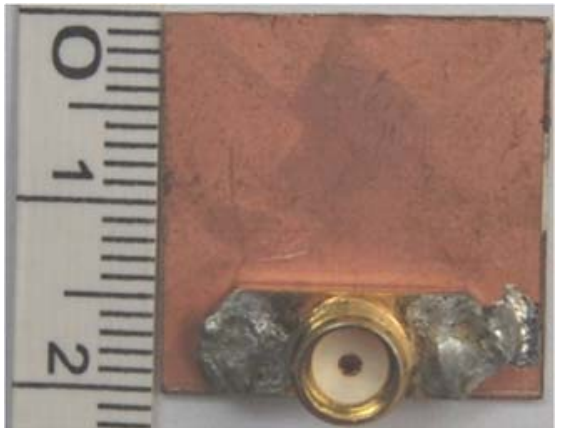

(b)

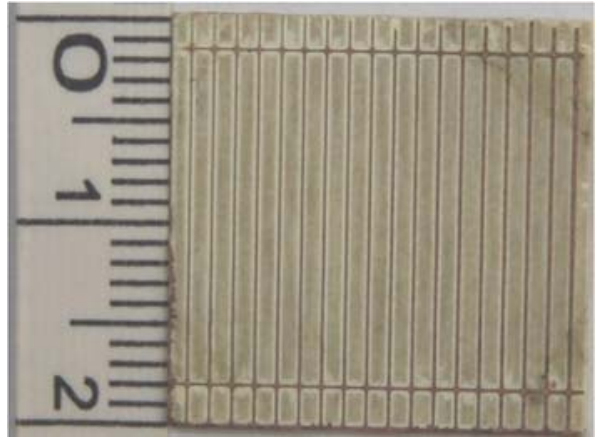

(d)

Fig. 3. Prototypes: (a) top view of patch 1, (b) bottom view of patch 1, (c) layer 1 of MS, and (d) layer 2 of MS

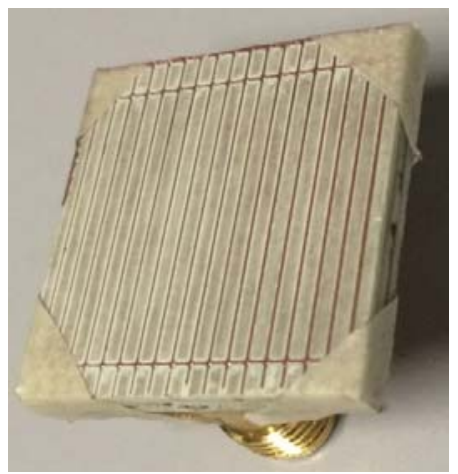

(a)

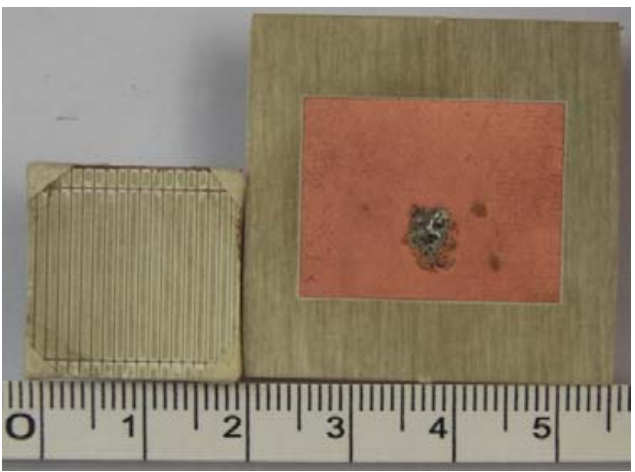

(b)

Fig. 4. Prototypes: (a) miniaturized-patch antenna (MS with patch 1), and (b) top view of miniaturized-patch antenna (left) together with patch 2 (right).

\section{Results and Discussions}

The objective of the study is to reduce the size of the patch antenna without degrading the performance. Thus, in this section, we compare the bandwidth, efficiency and radiation pattern of the miniaturized-patch antenna with those of patch 2 . In addition, we also show the 
performance of patch 1 with discussions.

\section{A. Refelection Coefficient S11}

The simulated and measured reflection coefficients S11 of patch 1 with and without using the MS are shown in Fig. 5. It can be seen that, without using the MS, the resonant frequency of patch 1 is $8 \mathrm{GHz}$. With the use of the MS, the resonant frequency is shifted down to $4 \mathrm{GHz}$, with a bandwidth of 3.96-4.05 GHz for $\mathrm{S} 11<-10 \mathrm{~dB}$. The frequency shift from 8 to $4 \mathrm{GHz}$ can be translated into an approximate reduction of more than $50 \%$ in dimensions or $75 \%$ in area, which is resulted from using the MS.

The S11 of patch 2 is also shown in Fig. 5 for comparison. It can be seen that patch 2 also has a resonant frequency of $4 \mathrm{GHz}$ with a bandwidth of 3.96-4.05 GHz, same as patch 1 using the MS. However, patch 2 occupies a much larger area of $35 \times 35 \mathrm{~mm}^{2}=1225 \mathrm{~mm}^{2}$. Thus, the MS can be used to reduce the required area of patch antenna by $67 \%$, yet having the similar resonant frequency and bandwidth.

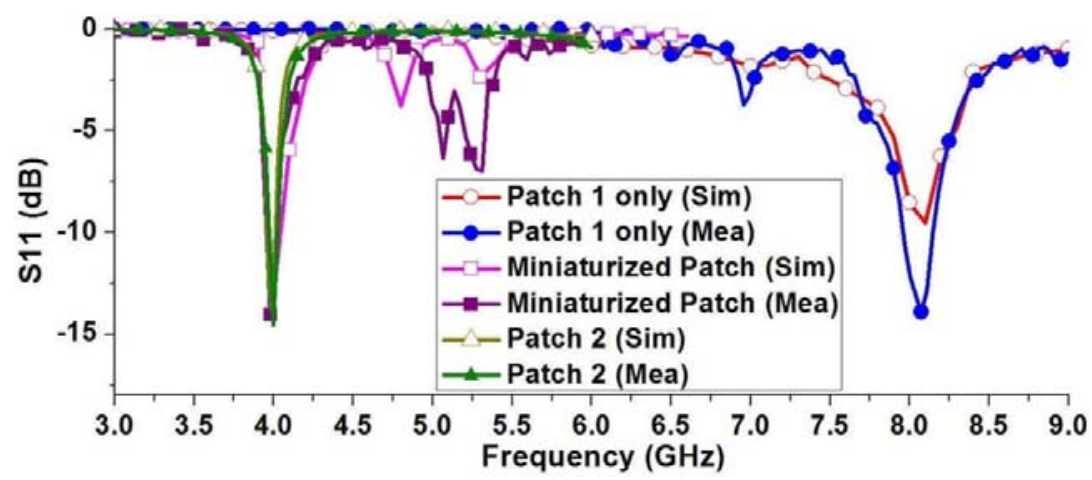

Fig. 5. Simulated and measured S11 of patch 1 with and without MS and patch 2

\section{B. Efficiency}

The results on efficiencies of the miniaturized-patch antenna and patch 2 are shown in Figs. 6(a) and (b), respectively. It can be seen that the miniaturized-patch antenna and patch 2 have the maximum efficiencies of $84 \%$ and $80 \%$, respectively, at $4 \mathrm{GHz}$. Thus the miniaturized-patch antenna has a higher efficiency than patch 2, but occupies a much smaller size. The simulated and measured results for patch 2 shown in Fig. $6(\mathrm{~b})$ agree very well. However, this is not the case for the miniaturized-patch as shown in Fig. 6(a). There is a large discrepancy between the simulated and measured results. The measured efficiency in Fig. 6(a) shows that the miniaturized-patch antenna has an efficiency of only $53 \%$ at $4 \mathrm{GHz}$, much lower than the simulated efficiency of $80 \%$ and also the measured efficiency of $75 \%$ for patch 2 . The large discrepancy is mainly due to cable effects caused by the feeding cable used in the antenna measurement equipment, the Starlab system [20]. For antennas with small ground planes, the cable effects could make the measured efficiency and realized gain much lower than those of simulation. The reason can be explained as follows. In simulation, there is no feeding cable used to feed the antenna. However, when an antenna is measured using the antenna measurement system, a coaxial feeding cable is needed to connect the antenna to the measurement system. 
For antennas with electrically small ground planes as in our miniaturized-patch antenna, current will flow back to the outer surface of the feeding cable, resulting in radiation [20, 21, 24] and affecting the measured radiation pattern. To reduce the cable effects on radiation pattern measurement, the feeding cable provided by Satimo for use with the Starlab system is covered with EM suppressant tubing to absorb radiation from the cable. This makes the shape of the antenna pattern measurement more accurate. However, as some radiation is absorbed by the EM suppressant tubing and does not get measured by the measurement system, the measured efficiency and gain will be smaller than the actual values, as shown in Figs. 6(a) and (b). Since the miniaturized-patch antenna has a much small ground plane than patch 2 , the reductions in efficiency and gain due to cable effects are more significant. To study the cable effects on measured results, the cable model developed in [20], as shown in the simulation model of Fig. 7, is included in simulation. The discrepancy between the simulated efficiencies with and without using the cable model is caused by the cable effects and can be used to remove the cable effects in real measurements. The measured efficiencies of the miniaturized-patch antenna and patch 2, after removing the cable effects as described, are also shown in Fig. 6 for comparison. It can be seen in Fig. 6(a) that, after removing the cable effects, the measured efficiency $(71.6 \%$ at $4 \mathrm{GHz})$ of the miniaturized-patch antenna has a much better agreement with the simulated efficiency (80\%) without using the cable model. Due to the larger ground plane used, patch 2 has much smaller cable effects, as shown in Fig. 6(b). It also can be seen that, after removing the cable effects, the measured efficiency of patch 2 is slightly increased from $75 \%$ to $78 \%$, closer to the simulated efficiency of $84 \%$ without the cable model. It should be pointed out that when an antenna is installed in practice, there is no cable used and so cable effects is not a problem.

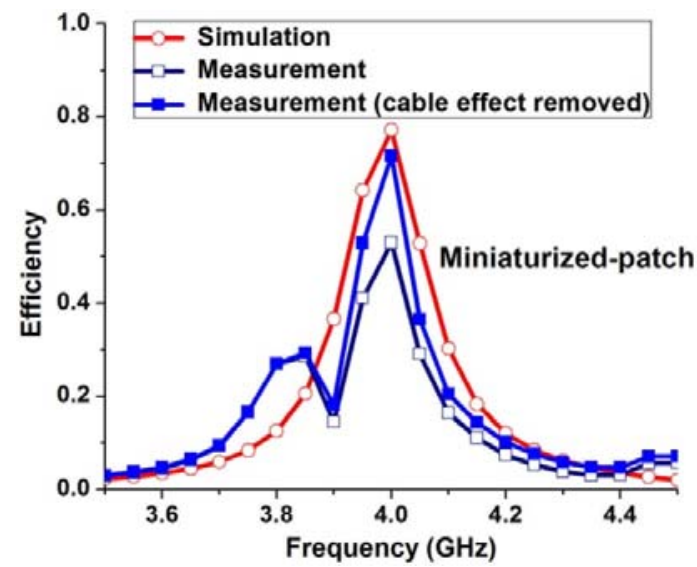

(a)

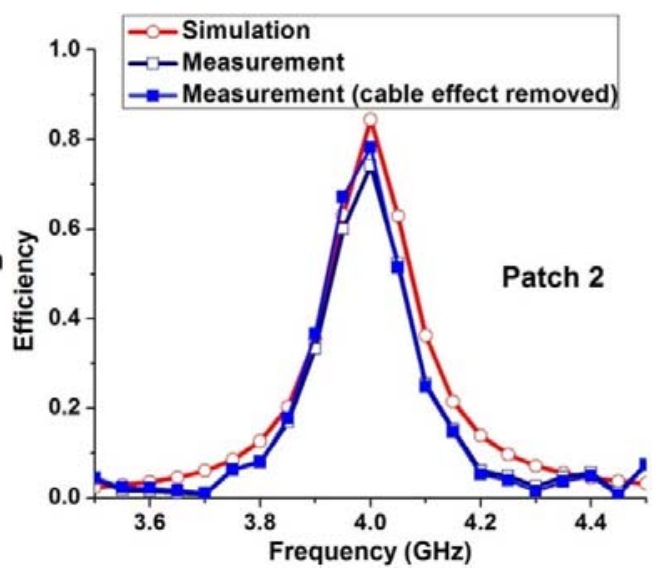

(b)

Fig. 6 Simulated and measured efficiencies of miniaturized-patch antenna and patch antenna 1 and b) patch antenna 2 with and with cable model. 


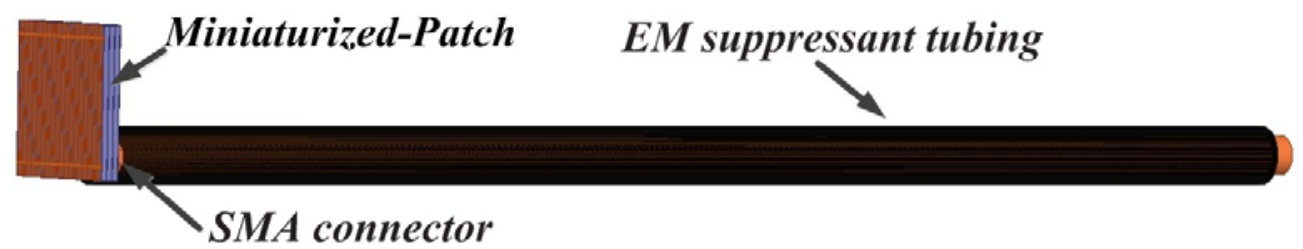

Fig. 7 Cable model

C. Realized Boresight Gain

The simulated and measured realized boresight gains of the miniaturized-patch antenna and patch 2 are shown in Figs. 8. The miniaturized-patch antenna and patch 2 have the simulated realized boresight gains of $5.1 \mathrm{dBi}$ and $5.2 \mathrm{dBi}$, respectively, at $4 \mathrm{GHz}$ as shown in Fig. 8. Due to the cable effects, the measured realized boresight gain of the miniaturized-patch antenna is only $2.47 \mathrm{dBi}$ at $4 \mathrm{GHz}$, significantly lower than the simulated result of $5.1 \mathrm{dBi}$. The measured boresight gain of patch 2 is $4.35 \mathrm{dBi}$ at $4 \mathrm{GHz}$. With the use of the cable model shown in Fig. 7, the measured realized gains of the antennas after removing the cable effects is also shown in Fig. 8. It can be seen that the simulation and measurement have much better agreements with each other. The measured realized boresight gain of the miniaturized-patch antenna is $4.95 \mathrm{dBi}$ at $4 \mathrm{GHz}$, even higher

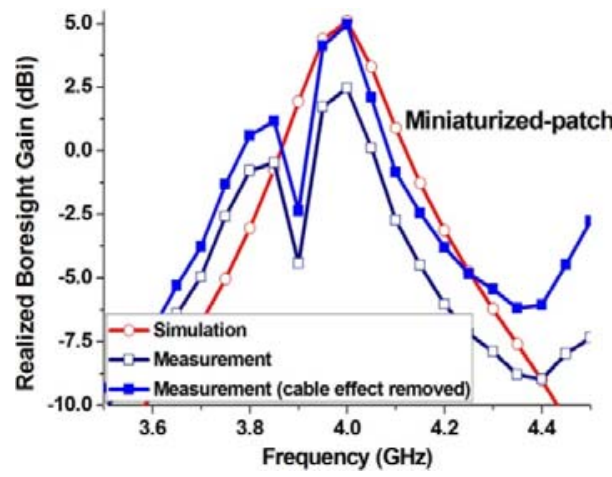

(a)

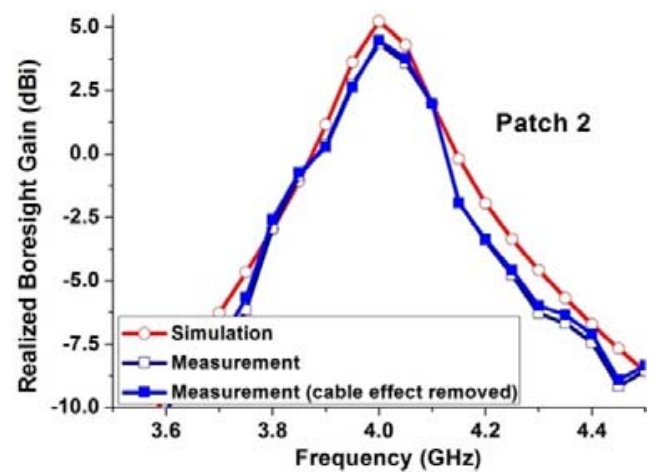

(b)

Fig. 8 Realized boresight gains of (a) miniaturized-patch antenna and (b) patch 2.

\section{Radiation Pattern}

The simulated and measured radiation patterns of the miniaturized-patch antenna and patch 2 at $4 \mathrm{GHz}$ in the $x-z$ and $x-y$ planes are shown in Figs. 9. For comparison, the measured results after removing the cable effects are also shown in the same figures, indicating that better agreements are observed particularly for the miniaturized-patch antenna. Fig. 9 shows that both the miniaturized-patch antenna and patch 2 have unidirectional radiation patterns in both the $x-y$ and $x-z$ planes, like typical patch antenna. 


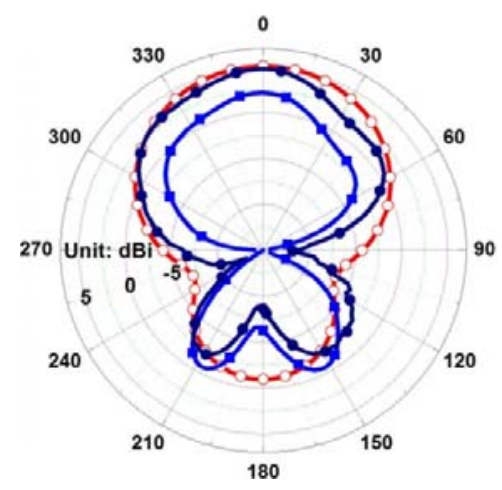

(a) $x z$-plane

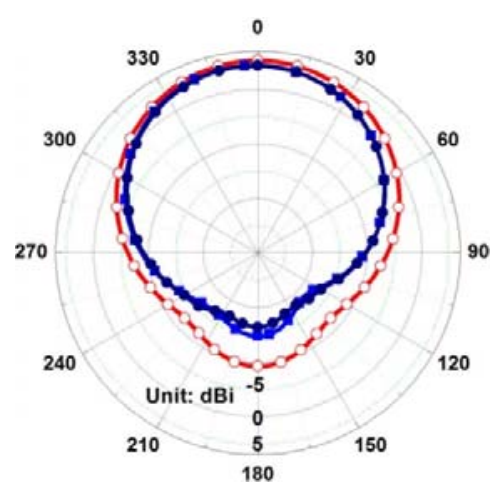

(c) $x z$-plane

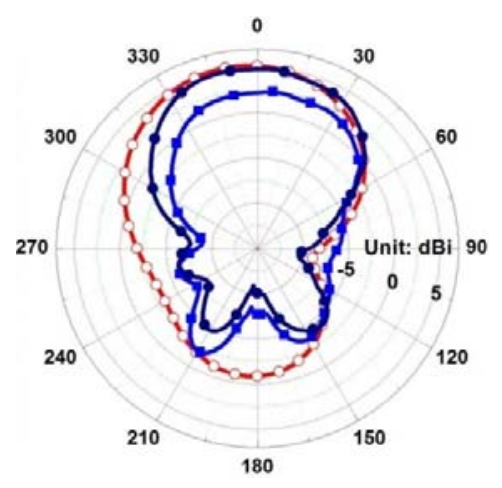

(b) yz-plane

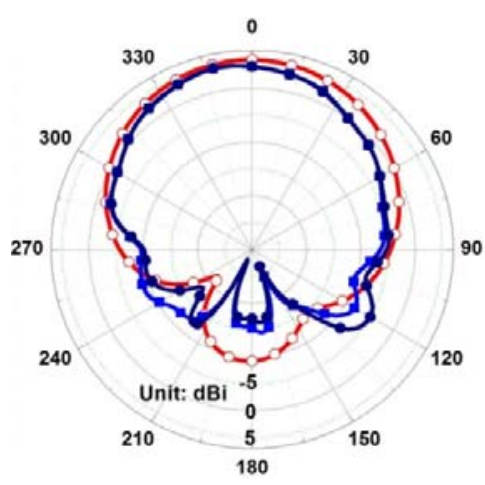

(d) yz-plane

Fig. 9 Radiation patterns of (a)\&(b) miniaturized-patch antenna and (c)\&(d) patch 2 at $4 \mathrm{GHz}$, $\rightarrow$ : simulation, $\rightarrow$ : measurement, $\longrightarrow$ - measurement with cable effect removed.

\section{Conclusions}

Using MS to miniaturize the size of a patch antenna has been proposed. The MS consists of two layers having patterns complementary to each other on the surfaces. The MS and the patch antenna are put in direct contact, leading to a very low profile. Simulated and measured results have shown, the MS can reduce the size of the patch antenna by $67 \%$, yet having the similar performances in terms of operating frequency, operating bandwidth and realized boresight gain.

\section{References}

[1] R. W. Ziolkowski, T. Ming-Chun, and Z. Ning, "An efficient, electrically small antenna with large impedance bandwidth simultaneously with high directivity and large front-to-back ratio," in Electromagnetic Theory (EMTS), Proceedings of 2013 URSI International Symposium on, 2013, pp. 885-887.

[2] R. W. Ziolkowski, T. Ming-Chun, and Z. Ning, "Progress towards an electrically small antenna with high efficiency and large bandwidth simultaneously with high directivity and a large front-to-back ratio," in Antenna Technology (iWAT), 2013 International Workshop on, 2013, pp. 175-178.

[3] B. Sentucq, A. Sharaiha, and S. Collardey, "Superdirective metamaterial-inspired electrically small antenna arrays," in Antennas and Propagation (EUCAP), 2013 7th European Conference on, 2013, pp. 151-155. 
[4] I. T. Nassar and T. M. Weller, "An electrically-small, 3-D cube antenna fabricated with additive manufacturing," in Silicon Monolithic Integrated Circuits in RF Systems (SiRF), 2013 IEEE 13th Topical Meeting on, 2013, pp. 162-164.

[5] N. Behdad, L. Meng, and Y. Yusuf, "A Very Low-Profile, Omnidirectional, Ultrawideband Antenna," Antennas and Wireless Propagation Letters, IEEE, vol. 12, pp. 280-283, 2013.

[6] X. L. Sun, S. W. Cheung, and T. I. Yuk, "Design of compact antenna for 2.4/4.9/5.2/5.8-GHz WLAN," Microwave and Optical Technology Letters, vol. 56, pp. 1360-1366, 2014.

[7] L. Li, C. Sing Wai, and Y. Tung Ip, "Compact multiple-input-multiple-output antenna using quasi-self-complementary antenna structures for ultrawideband applications," Microwaves, Antennas \& Propagation, IET, vol. 8, pp. 1021-1029, 2014.

[8] X. L. Sun, S. W. Cheung, and T. I. Yuk, "Dual-Band Monopole Antenna with Compact Radiator for 2.4/3.5 GHz WiMAX Applications," Microwave and Optical Technology Letters, vol. 55, pp. 1765-1770, 2013.

[9] J. D. Kraus and R. J. Marhefka, Antennas: For All Applications, Third Edition, 2011.

[10] C. A. Balanis, ANTENNA THEORY - ANALYSIS AND DESIGN. Hoboken, New Jersey, US: John Wiley \& Sons, Inc., 2005.

[11] C. L. Holloway, E. F. Kuester, J. A. Gordon, J. O"Hara, J. Booth, and D. R. Smith, "An Overview of the Theory and Applications of Metasurfaces: The Two-Dimensional Equivalents of Metamaterials," Antennas and Propagation Magazine, IEEE, vol. 54, pp. 10-35, 2012.

[12] H. L. Zhu, S. W. Cheung, K. L. Chung, and T. I. Yuk, "Linear-to-Circular Polarization Conversion Using Metasurface," Antennas and Propagation, IEEE Transactions on, vol. 61, pp. 4615-4623, 2013.

[13] H. L. Zhu, S. W. Cheung, X. H. Liu, Y. F. Cao, and T. I. Yuk, "Frequency reconfigurable slot antenna using metasurface," in Antennas and Propagation (EuCAP), 2014 8th European Conference on, 2014, pp. 2575-2577.

[14] H. L. Zhu, S. W. Cheung, X. H. Liu, and T. I. Yuk, "Design of Polarization Reconfigurable Antenna using Metasurface," Antennas and Propagation, IEEE Transactions on, vol. PP, pp. 1-1, 2014.

[15] H. L. Zhu, K. L. Chung, X. L. Sun, S. W. Cheung, and T. I. Yuk, "CP metasurfaced antennas excited by LP sources," in Antennas and Propagation Society International Symposium (APSURSI), 2012 IEEE, 2012, pp. 1-2.

[16] H. L. Zhu, X. H. Liu, S. W. Cheung, and T. I. Yuk, "Frequency-Reconfigurable Antenna Using Metasurface," Antennas and Propagation, IEEE Transactions on, vol. 62, pp. 80-85, 2014.

[17] K. L. Chung and S. Chaimool, "Diamagnetic metasurfaces for performance enhancement of microstrip patch antennas," in Antennas and Propagation (EUCAP), Proceedings of the 5th European Conference on, 2011, pp. 48-52.

[18] H. L. Zhu, S. W. Cheung, and T. I. Yuk, "Antenna reconfiguration using metasurfaces," in The 35th Progress In Electromagnetics Research Symposium (PIERS 2014) Guangzhou (Canton), China, 2014 , pp. p. $2400-2404$

[19] L. Li, S. W. Cheung, and T. I. Yuk, "Compact MIMO Antenna for Portable Devices in UWB Applications," Antennas and Propagation, IEEE Transactions on, vol. 61, pp. 4257-4264, 2013.

[20] L. Liu, S. W. Cheung, Y. F. Weng, and T. I. Yuk, Cable Effects on Measuring Small Planar UWB Monopole Antennas, 2012.

[21] L. Liu, S. W. Cheung, T. I. Yuk, and D. Wu, "A compact ultrawideband MIMO antenna," in Antennas and Propagation (EUCAP), 2013 7th European Conference on, 2013, pp. 2108-2111. 
[22] X. L. Sun, S. W. Cheung, and T. I. Yuk, "Dual-Band Monopole Antenna With Frequency-Tunable Feature for WiMAX Applications," Antennas and Wireless Propagation Letters, IEEE, vol. 12, pp. 100-103, 2013.

[23] X. L. Sun, S. W. Cheung, and T. I. Yuk, "Frequency-Tunable Monopole Antenna for Wimax Applications," Microwave and Optical Technology Letters, vol. 55, pp. 1902-1907, 2013.

[24] S. Xiao Lei, L. Li, S. W. Cheung, and T. I. Yuk, "Dual-Band Antenna With Compact Radiator for 2.4/5.2/5.8 GHz WLAN Applications," Antennas and Propagation, IEEE Transactions on, vol. 60, pp. 5924-5931, 2012. 